\title{
sd-Shell Study with a Multi-Configuration Mixing Approach designed for Large Scale Nuclear Structure Calculations
}

\author{
E. Bender, K.W. Schmid and Amand Faessler \\ Institut für Theoretische Physik \\ Universität Tübingen \\ Auf der Morgenstelle 14 \\ D-72076 Tübingen \\ Germany
}

\begin{abstract}
A systematic numerical investigation of a recently developed nuclear structure approach is presented which diagonalizes the Hamiltonian in the space of the symmetry-projected Hartree-Fock-Bogoliubov (HFB) vacuum and symmetry-projected quasiparticle excitations with respect to it. The underlying HFB transformation, which is assumed to be time-reversal and axially symmetric, is determined by variation after the projection. The model allows the use of large basis systems. It has been applied to the calculation of energy spectra of several even-even, odd-odd and odd mass nuclei in the $s d$ shell with mass numbers reaching from $A=20$ to 30 . The Chung-Wildenthal interaction has been used. Good agreement with the exact shell model diagonalization and a considerable improvement on a previous approach, where the HFB transformation was significantly more restricted, is obtained.
\end{abstract}




\section{Introduction}

Microscopic nuclear structure models try to explain the excitation energies and other experimental spectroscopic information starting from a realistic effective nucleonnucleon interaction. An example is the interacting shell model. It is generally acknowledged to be the most fundamental theory of a nucleus [1] [2] [3]. But since the number of A-nucleon configurations to be diagonalized increases rapidly with the number of single particle states, its application is limited to rather small basis systems, like the $s d$ shell [4]. Thus for many problems the configuration spaces have to be truncated drastically. How to select a numerically feasible number of physical relevant configurations is the central question of all nuclear structure models which use the shell model as the conceptual basis.

One prescription for such a truncation is provided by the mean field approaches like the Hartree-Fock [5] [6] or the Hartree-Fock-Bogoliubov (HFB) [7] [8] theory. They use the variational principle to describe the ground state of a nucleus by one single Slater determinant. However, usually in this determinant all physical symmetries are broken. Thus one has to use projection techniques to find the component with the desired quantum numbers in order to obtain physical states. Moreover, in order to obtain the energetically lowest solution for a given set of quantum numbers, one has to perform the projection on the good symmetries before the mean field is determined by variation.

Following this idea, a whole hierarchy of symmetry conserving mean field theories and their extensions into multi-configuration mixing methods have been proposed by some of our group a few years ago [9]. They are known as the VAMPIR (Variation After Mean field Projection In Realistic model spaces) and the MONSTER (MOdel for handling many Number- and Spin-projected Two-quasiparticle Excitations with Realistic interactions and model spaces) approaches.

The models of the VAMPIR family, like VAMPIR [10, EXCITED VAMPIR [1], FED (FEw Determinant) VAMPIR and EXCITED FED VAMPIR [12], can be used to describe the lowest few states of a given symmetry, irrespective of their particular structure. Each state is obtained by variational calculations. So, e.g., the VAMPIR model approximates the lowest state of certain quantum numbers by just a single symmetry-projected HFB vacuum. The underlying HFB transformation is determined by variation after the projection on the desired quantum numbers. Correlating configurations as well as excited states can be obtained by chains of similar variational calculations.

On the other hand, the MONSTER approaches are constructed for the description of complete excitation spectra with respect to a one-body transition operator 44 
[13]. Thus they are well suited, e.g., for the description of giant resonances. States which are reached by a one-body operator are of similar structure as the initial ground or yrast state. Therefore they can be described by expanding the nuclear wavefunction around a symmetry-projected reference vacuum, which may be either a usual HFB, or, e.g., a VAMPIR solution for the ground or yrast state. This is done in the MONSTER approach. More explicitly, the MONSTER configuration space is built out of the symmetry-projected vacuum and all symmetry-projected two-quasiparticle excitations defined on it. The excited states are then obtained by diagonalizing the residual interaction between these configurations.

In all realistic applications up to now, these models have been simplified by imposing symmetry restrictions on the underlying HFB transformations. In the first VAMPIR calculations only real, time-reversal invariant and axially symmetric HFB transformations, which neither mix proton and neutron states nor states of different parity, were admitted [10] [11]. With this real VAMPIR approach, as it is called, only states in even-even nuclei with even spin and positive parity can be described. If a MONSTER calculation is based on such a real VAMPIR transformation, states with different symmetries are described entirely by the two-quasiparticle components of the wavefunction. Some years ago, this VAMPIR approach has been improved by using HFB transformations, which are essentially complex and allow proton-neutron and parity mixing [14]. Only time reversal and axiality are still imposed on the transformation. This complex VAMPIR approach can describe states of arbitrary spin parity in even-even and odd-odd nuclei. It considers many more nucleon correlations [15].

Recently the MONSTER approach has been generalized for the use of such complex VAMPIR transformations [16]. It was tested for two light even-even $s d$ shell nuclei. The results were encouraging. The performance of the complex MONSTER description for heavier even-even nuclei, for odd-odd and odd-mass nuclei, however, remained an open question. In the present paper we try to answer this question by a systematic investigation.

For this purpose the complex MONSTER model was applied to several even-even, odd-odd and odd mass systems. The calculations were performed again in an $s d$ shell basis, in order to be able to compare with the exact shell model results. The considered nuclei of each type are covering the range from very light systems to the ones with the biggest possible numbers of shell model configurations in the $s d$ shell. The results are compared to those of the older more restricted real MONSTER approach, too. It is demonstrated that the complex MONSTER approach approximates the shell model results very well and shows a clear improvement on the older real approach.

In the next section the main features of the MONSTER on VAMPIR model are 
summarized and the consequences of the symmetry restrictions for the HFB transformation are shortly explained. In section (3) the results of the application are presented and discussed. Finally conclusions and an outlook are given in (4).

\section{The Model}

The detailed formulation of the MONSTER on VAMPIR approach has been given elsewhere [16]. Therefore only the main ideas of the model are sketched here.

\subsection{The VAMPIR model}

A finite model space is defined by a $D$-dimensional set of orthonormal single particle wave functions, which are eigenstates of some spherically symmetric one-body potential, e.g. the harmonic oscillator. In second quantization the corresponding creation and annihilation operators are denoted by $\left\{c_{i}^{\dagger}, c_{k}^{\dagger}, \ldots\right\}_{D}$ and $\left\{c_{i}, c_{k}, \ldots\right\}_{D}$, respectively. The indices $i, k$ summarize the quantum numbers of a state. It is assumed that the effective many body Hamiltonian appropriate for this model space is known and can be represented by a sum of only one- and two-body terms

$$
\hat{H}=\hat{T}+\hat{V} .
$$

The one-body part $\hat{T}$ contains the matrix elements of the kinetic energy (or, if an inert core is assumed, the single particle energies) and the two-body part $\hat{V}$ the matrix elements of the effective interaction.

Starting from the chosen particle basis, the Hartree-Fock-Bogoliubov (HFB) transformation [8] is used to define the corresponding quasiparticle basis with its quasiparticle creation and annihilation operators, $\left\{a_{\alpha}^{\dagger}, a_{\beta}^{\dagger}, \ldots\right\}_{D}$ and $\left\{a_{\alpha}, a_{\beta}, \ldots\right\}_{D}$. $\alpha, \beta$ enumerate the quasiparticle states. The HFB transformation is the most general linear transformation which ensures that the Fermion anti-commutation relations are fulfilled by the quasiparticle operators. In matrix notation it is given by

$$
\left(\begin{array}{c}
a^{\dagger}(F) \\
a(F)
\end{array}\right)=F\left(\begin{array}{c}
c^{\dagger} \\
c
\end{array}\right)
$$

with $F$ being a unitary $2 D \times 2 D$ dimensional matrix. The vacuum $|F\rangle$ for a set of quasiparticles obtained by the HFB transformation $F$ is defined in the usual way by the request that application of any quasiparticle annihilation operator onto it should yield zero,

$$
a_{\alpha}(F)|F\rangle=0 \quad \forall \alpha=1, \ldots, D .
$$


An $n$-quasiparticle state with respect to the vacuum is defined by

$$
\left|F\left\{a^{\dagger}\right\}_{n}\right\rangle=\left(\prod_{\alpha=1}^{n} a_{\alpha}^{\dagger}(F)\right)|F\rangle \quad \text { for } \quad n=1, \ldots, D \text {. }
$$

The vacuum as well as the $n$-quasiparticle states contain components of many nucleon configurations with different angular momenta, different angular momentum $z$-components, both parities and various proton and neutron numbers [17. In order to get physical states which are characterized by good quantum numbers, projection techniques have to be used for selecting the components with the desired symmetries. The corresponding projection operator $\hat{\Theta}_{M K}^{A T_{z} I^{\pi}}$ is a product of projection operators,

$$
\hat{\Theta}_{M K}^{A T_{z} I^{\pi}} \equiv \hat{P}(I M ; K) \hat{P}\left(2 T_{z}\right) \hat{P}(A) \hat{P}(\pi),
$$

where the first projects on good angular momentum $I$ with $z$-component $M$, the next ones on good isospin $z$-component $T_{z}$, good mass number $A$ and finally on good parity $\pi$. Explicit expressions for them can be found in [14]. All projectors apart from $\hat{P}(\pi)$ are integral operators.

Physical configurations are obtained by applying the operator $\hat{\Theta}_{M K}^{A T_{z} I^{\pi}}$ on the above introduced quasiparticle configurations. Since any of the $n$-quasiparticle configurations $\left|F\left\{a^{\dagger}\right\}_{n}\right\rangle$ built on the vacuum $|F\rangle$ can be looked upon at the same time as a vacuum for another HFB transformation $F^{\prime}$, it is enough to consider only HFB vacua in the following. Thus, a physical configuration with a good symmetry $S$, where $S$ represents the quantum numbers $A T_{z} I^{\pi}$, is given by

$$
|F ; S M\rangle=\sum_{K=-I}^{I} \hat{\Theta}_{M K}^{S}|F\rangle f_{K}^{S}
$$

The sum over all intrinsic angular momentum $z$-components $K$ has to be taken in order to avoid a dependency of the projected wave function on the orientation of the intrinsic reference frame [9].

In the VAMPIR approach one symmetry-projected vacuum (6) is used to describe the energetically lowest state of a given symmetry $S$. The configuration mixing degrees of freedom $f_{K}^{S}$ and the underlying HFB transformation $F$ are determined by variation

$$
\delta E^{S} \equiv \delta \frac{\langle F ; S M|\hat{H}| F ; S M\rangle}{\langle F ; S M \mid F ; S M\rangle}=0 .
$$

This variation leads to three sets of equations which have to be solved self-consistently [14]. One obtains the optimal description of the considered yrast state which can be achieved by using only a single determinant. Generally this VAMPIR approach yields already a rather good approximation to the actual state [15]. 
A similar approach, the FED VAMPIR, uses a linear combination of a few symmetryprojected vacua to describe one yrast state. Here, the correlating configurations, which contribute to one state, are searched for by successive variational calculations [12]. Furthermore, by introducing orthogonality constraints, the VAMPIR and the FED VAMPIR procedures can be easily extended to the description of excited states. These EXCITED VAMPIR [14] and EXCITED FED VAMPIR [12] models can describe states of any arbitrary structure. But since each state is searched for by variation, a numerical application is quite time consuming. Thus the VAMPIR models are especially well suited for the description of the lowest few states of a given symmetry $S$.

For problems which require a complete excitation spectrum with respect to some transition operator, it is better to follow another avenue. Since here only specific configurations are needed, one can describe them by expanding the nuclear wave functions around a suitable VAMPIR vacuum. This is explained in the next section.

\subsection{The MONSTER on VAMPIR approach}

For states with symmetry $S$ the configuration space is chosen to consist of the symmetry-projected vacuum

$$
|F ; S M K\rangle \equiv \hat{\Theta}_{M K}^{S}|F\rangle
$$

and the symmetry-projected two-quasiparticle states with respect to it

$$
|F \alpha \beta ; S M K\rangle \equiv \hat{\Theta}_{M K}^{S} a_{\alpha}^{\dagger}(F) a_{\beta}^{\dagger}(F)|F\rangle .
$$

The underlying HFB transformation $F$ is fixed in a preceding VAMPIR calculation for an yrast state, which not necessarily needs to have just the same symmetry $S$. In many cases it is sufficient to use the HFB transformation obtained for the ground state of the considered nucleus.

A general wavefunction for excited states is then given by

$$
\left|\psi_{i}(F) ; S M\right\rangle=\left\{\sum_{K=-I, \ldots,+I}|F ; S M K\rangle g_{0 K ; i}^{S}+\sum_{\substack{\alpha<\beta \\ K=-I, \ldots,+I}}|F \alpha \beta ; S M K\rangle g_{\alpha \beta K ; i}^{S}\right\} .
$$

The expansion coefficients $g^{S}$ are obtained by diagonalizing the effective Hamiltonian in the space of the nonorthogonal configurations (8, 9).

The above choice of the model space restricts the MONSTER on VAMPIR approach to excited states with a structure similar to that of the underlying projected vacuum. 
This is here desired, since we are interested in states predominantly populated by a transition from the vacuum via a one-body operator. Furthermore it should be mentioned that the MONSTER approach provides an approximate elimination of spurious center-of-mass excitations, which can be introduced by the configuration mixing if more than one major oscillator shell is taken as particle basis (for details see [16]).

\subsection{Symmetry restrictions imposed on the HFB transformation}

If the most general HFB transformations are allowed, symmetry-projected vacua of type (6) can describe arbitrary states in arbitrary nuclei [14. Up to now, however, this has not been achieved in any numerical implementation. Instead, for the existing numerical realizations of the VAMPIR approaches, certain symmetry requirements were imposed on the underlying HFB transformations.

In the first VAMPIR calculations [10] axial symmetry and time-reversal invariance were assumed, parity and proton-neutron mixing were neglected and only real HFB transformation coefficients were admitted. As a consequence of these approximations the real VAMPIR approach was only suitable for positive parity states with even angular momenta in doubly even nuclei. Performing MONSTER calculations on top of such a real VAMPIR solution these limitations are removed. In the twoquasiparticle approximation not only states with arbitrary spin-parity in doubly even but also in doubly odd systems become accessible. However, for the description of odd spin and/or negative parity states in doubly even nuclei one has to use mean fields obtained for different spin and/or parity values than the considered one. And for doubly odd systems one even has to rely on mean fields for neighbouring systems. Considering as configuration space for the MONSTER calculation all symmetryprojected one-quasiparticle states

$$
|F \alpha ; S M K\rangle \equiv \hat{\Theta}_{M K}^{S} a_{\alpha}^{\dagger}(F)|F\rangle
$$

arbitrary states in odd systems become approachable, too. Here again, one has to build the one-quasiparticle spectrum on the mean field determined for a neighbouring doubly even nucleus.

In the more recent implementations of the VAMPIR calculations parity as well as proton-neutron mixing are taken into account and essentially complex HFB transformations are admitted. In this complex VAMPIR approach only axial symmetry and time reversal invariance are kept [14]. This introduces many more correlations into the projected vacua than the older real calculations. Now states of arbitrary 
spin-parity in both doubly even and doubly odd systems are accessible. The complex vacua contain all possible two nucleon couplings, but some four-, six- and more nucleon couplings are missing [15]. Only recently the MONSTER approach could be extended to such complex VAMPIR vacua [16]. Here, in addition to doubly even nuclei, also the calculation for doubly odd nuclei can be based on a HFB transformation determined for just the considered system itself. And the structures which are missing in the complex VAMPIR vacuum can be introduced by the two-quasiparticle admixtures. However, for the description of odd systems one still has to use the onequasiparticle approximation like in the older real approach. But now aside from the mean fields of neighbouring even-even nuclei also the ones of neighbouring odd-odd nuclei can be chosen to construct the one-quasiparticle states (11).

Generally, since the underlying HFB transformation is much more general and the configuration space is much larger, the complex MONSTER is expected to yield a better description of nuclear spectra than the real one.

\section{$3 \quad$ A systematic application to $s d$ shell nuclei}

In [16] we presented as a first test of the new complex MONSTER approach an application to ${ }^{20} \mathrm{Ne}$ and ${ }^{22} \mathrm{Ne}$. Considering the $s d$ shell as model space, it was shown that the model is able to reproduce the shell model spectrum of ${ }^{20} \mathrm{Ne}$ exactly. For ${ }^{22} \mathrm{Ne}$ it yielded an excellent approximation to the shell model result. A clear improvement on the older real approach was found. However, the two investigated nuclei are even-even systems. Moreover, they are quite light : for both nuclei the number of shell model states with a certain spin is comparable to the number of complex MONSTER configurations. How does the approach perform for heavier nuclei, where the number of states is much larger than the number of complex MONSTER configurations ? Furthermore, which kind of agreement is obtained for odd-odd nuclei and for odd mass systems, where the simpler one-quasiparticle description is used ? These questions are adressed in the present investigation.

Again the $1 s 0 d$ shell was chosen as single particle basis in order to be able to compare with exact shell model configuration mixing calculations. The single particle energies $\epsilon(d 5 / 2)=-4.15 \mathrm{MeV}, \epsilon(s 1 / 2)=-3.28 \mathrm{MeV}$, and $\epsilon(d 3 / 2)=+0.93 \mathrm{MeV}$ for both protons and neutrons [18] and the effective two-body residual interaction (the mass-

dependent version of the Chung and Wildenthal force 19 $\left(\hat{V}(A)=\hat{V}(18) \times(18 / A)^{\alpha}\right)$ with $\alpha=1 / 3$ instead of $\alpha=0.3)$ are the same as in our earlier calculations. This force is generally accepted to be "the standard" force for the $s d$ model space. The results are compared in addition to those obtained by the more restricted real MONSTER approach. 


\subsection{Even-even nuclei}

As examples for "heavier" even-even $s d$-shell nuclei, we calculated the spectra of ${ }_{12}^{24} \mathrm{Mg},{ }_{12}^{26} \mathrm{Mg},{ }_{14}^{28} \mathrm{Si}$ and ${ }_{14}^{30} \mathrm{Si}$ within the complex and the real MONSTER approaches. For each MONSTER calculation the underlying HFB transformation was determined by a preceding VAMPIR calculation for the $0^{+}$-ground state. This HFB transformation $\mathrm{F}\left(0^{+}\right)$has then been used for states of all spins in one nucleus.

The energies obtained by the complex and the more restricted real MONSTER have been compared to those from an exact shell model diagonalization. The result of this investigation is summarized in table 1 . The average deviation of the energies of the yrast states with spin $0^{+}$to $5^{+}$from the shell model energies is presented for each nucleus. The deviation is given in $\mathrm{keV}$ and in percent of the shell model ground state energy of the nucleus referred to. The exact ground state energy is also displayed. For comparison the corresponding values for the nuclei ${ }^{20} \mathrm{Ne}$ and ${ }^{22} \mathrm{Ne}$ of our first application are listed here, too. The table shows furthermore the minimum and maximum number of shell model configurations per spin for each nucleus and the corresponding spin value. In addition the number of shell model states per spin averaged over the states with spin $0^{+}$to $5^{+}$is given. It should be compared to the number of MONSTER configurations available in the $s d$-shell. As explained in more detail in [16] the complex MONSTER provides 57 configurations for the description of spin $0^{+}$states, 151 for $1^{+}, 223$ for $2^{+}, 259$ for $3^{+}, 275$ for $4^{+}$, and always 277 for all higher spin states. In the more restricted real MONSTER approach less configurations per spin are available : 21 for the $I=0$ states, 30 for $I=1,61$ for $I=2,56$ for $I=3$, always 60 for $I=5,7,9,11,13$, and always 73 for the $I=4,6,8,10,12,14$ states, respectively.

As expected for both MONSTER approaches the mean deviation of the yrast state energies from the exact shell model result, averaged for spins $0^{+}$to $5^{+}$, does increase with the number of actually existing shell model states. For the complex MONSTER the largest deviation measured relatively to the shell model ground state energy occurs for ${ }^{26} \mathrm{Mg}$ and amounts to $1.43 \%$. For ${ }^{20} \mathrm{Ne}$ the complex calculation reproduced the exact result. In the real case the largest average deviation, $2.42 \%$, is found for ${ }^{24} \mathrm{Mg}$ and the smallest, $0.87 \%$, for ${ }^{22} \mathrm{Ne}$. For all nuclei the complex MONSTER approach yields a clear improvement on the real one. The largest energy gain could be achieved in ${ }^{24} \mathrm{Mg}$, where the averaged deviation of the real MONSTER is 3.8 times larger than the one of complex MONSTER. The smallest amount of energy was won for ${ }^{26} \mathrm{Mg}$, where the average deviation of the real approach is by a factor of 1.3 larger compared to the complex one. For all even-even nuclei the complex MONSTER approach yields an excellent approximation to the exact calculation.

As an example, in fig. 1 , the spectra of ${ }^{28} \mathrm{Si}$ as obtained by the complex and the real 
MONSTER are shown up to $\sim 14 \mathrm{MeV}$ above the shell model ground state energy. For comparison the energies resulting from the shell model diagonalization are plotted up to $\sim 12 \mathrm{MeV}$ excitation energy. It can be seen that the complex MONSTER reproduces the shell model spectrum well and shows a clear improvement on the real MONSTER : the states are considerably more bound and many more shell model states can be described.

The nucleus ${ }^{28} \mathrm{Si}$ was investigated in more detail, since it has the largest number of states of all $s d$-shell nuclei. There exist in total 93710 states. More precisely, there are $3372 I^{\pi}=0^{+}$states, $9216 I^{\pi}=1^{+}, 13562 I^{\pi}=2^{+}, 15385 I^{\pi}=3^{+}, 15089 I^{\pi}=4^{+}$, $12876 I^{\pi}=5^{+}$and $9900 I^{\pi}=6^{+}$states. For the higher spins the number of states per spin continues to decrease; there is just one state with the highest possible spin $I^{\pi}=14^{+}$. In fig. 2 the yrast state energies as obtained by various models of the VAMPIR and MONSTER family are compared for the spins $0^{+}$to $6^{+}$. The energies from the exact shell model calculation are given in the leftmost column. Then, from left to right, the energies calculated by the complex MONSTER, the complex VAMPIR, the real MONSTER, and the real VAMPIR are shown. Note that the real VAMPIR approach can only describe even spin states.

The average deviation of the energies obtained by the complex MONSTER approach from the shell model, now including the $6^{+}$yrast state, is about $1085 \mathrm{keV}$ or $0.79 \%$ of the shell model ground state energy of $137.495 \mathrm{MeV}$. The deviation for the even spin states is on average somewhat smaller, namely $840 \mathrm{keV}$, than the one for the odd spin states, which amounts to $1412 \mathrm{keV}$. This was found also for the other investigated even-even nuclei, apart from ${ }^{24} \mathrm{Mg}$, where the difference is insignificant. There the average deviation of the odd spin states was only $51 \mathrm{keV}$ larger than for the even spin states. Real MONSTER gives for ${ }^{28} \mathrm{Si}$ an average deviation of $1746 \mathrm{keV}$, which amounts to $1.27 \%$ of the shell model ground state energy.

As it is to be expected, the complex MONSTER yrast states are more bound than the ones calculated by complex VAMPIR, except for the $0^{+}$VAMPIR state, which is stable with respect to any projected two-quasiparticle excitations of the same symmetry. Obviously the odd spin states $1^{+}, 3^{+}$and $5^{+}$are not well described by the complex VAMPIR. When the difference between the binding energies obtained by the two models is so pronounced, it can be inferred that these state are dominated by the above mentioned "missing couplings", which are not accessible within the complex VAMPIR model. This was also found in Ref. [16], where the odd spin yrast states of ${ }^{22} \mathrm{Ne}$ were rather poorly described by the complex VAMPIR approach.

The differences in the binding energies of real MONSTER and real VAMPIR are negligibly small. The even spin real MONSTER yrast states, $2^{+}, 4^{+}$and $6^{+}$, are on average only $56 \mathrm{keV}$ more bound. The $0^{+}$state does not mix with the projected two-quasiparticle states of the same symmetry in the real case, too. 


\subsection{Odd-odd nuclei}

In order to study the quality of the complex MONSTER approach for applications to odd-odd nuclei, we calculated the energy spectra of ${ }_{9}^{20} \mathrm{~F},{ }_{11}^{22} \mathrm{Na},{ }_{11}^{24} \mathrm{Na},{ }_{13}^{26} \mathrm{Al},{ }_{13}^{28} \mathrm{Al}$ and ${ }_{15}^{30} \mathrm{P}$. For each nucleus the complex MONSTER configuration space was built on the HFB transformation, which yielded the energetically lowest VAMPIR solution in just this nucleus, no matter which spin it corresponded to. The spectra have also been calculated with real MONSTER. In this case one has to take the HFB transformation of a neighbouring even-even nucleus to construct the MONSTER configuration space. Naturally, one has several possibilities : for an odd-odd nucleus with $\mathrm{Z}$ protons and $\mathrm{N}$ neutrons one can take the even-even neighbours with the proton and neutron numbers $(\mathrm{Z}-1, \mathrm{~N}-1),(\mathrm{Z}-1, \mathrm{~N}+1),(\mathrm{Z}+1, \mathrm{~N}-1)$, and $(\mathrm{Z}+1, \mathrm{~N}+1)$. It is a priori not clear, which choice may be the best. Therefore we looked in each neighbour for the HFB transformation, which belongs to the $0^{+}$ground state VAMPIR solution and used each for a subsequent real MONSTER calculation.

The energies obtained by both MONSTER approaches have been compared to the ones of a full shell model configuration mixing calculation. To get a quantitative measure, for each nucleus as already for the even-even nuclei, the average deviation of the energies of the yrast states with spins $0^{+}$to $5^{+}$from the shell model energies has been calculated. The resulting deviations are shown in table 2, again in $\mathrm{keV}$ and in percent of the shell model ground state energy. Besides the energy the table displays the spin of the shell model ground state and, for complex MONSTER the spin of the VAMPIR solution which was used. For real MONSTER always the result for that spectrum which yielded the best agreement with the shell model is listed in the table. The corresponding neighbouring nucleus is indicated. The real MONSTER spectra for one odd-odd nucleus, obtained from the HFB transformations of the various possible neighbours, differ often quite much. So, e.g., for ${ }^{22} \mathrm{Na}$ the average deviation of the yrast states $0^{+}$to $5^{+}$is $4.85 \%$ if the HFB transformation for the $0^{+}$ VAMPIR solution of ${ }^{22} \mathrm{Ne}$ is used, and $1.47 \%$ for the one of ${ }^{20} \mathrm{Ne}$.

Table 2 also shows the minimum and maximum number of shell model configurations per spin and the number per spin averaged for spins $0^{+}$to $5^{+}$. Since protons and neutrons are mixed by the complex HFB transformation, the same configurations are used for the calculation of even-even and odd-odd nuclei in a complex MONSTER calculation. Thus the number of configurations contributing to one spin given in section (3.1) is valid here, too. For the real MONSTER with no proton-neutron mixing the situation is different compared to the even-even case. For the description of odd-odd nuclei in $s d$ shell there are 14 configurations available for the $0^{+}$states, 39 for $1^{+}, 57$ for $2^{+}, 67$ for $3^{+}, 71$ for $4^{+}$and always 72 for the states with spins $5^{+}, \ldots 13^{+}$. These numbers are comparable in size to the ones of the even-even case. 
As already for the even-even nuclei, also for the odd-odd ones an increase of the average deviation with the number of shell model states per spin is observed. For ${ }^{20} \mathrm{~F}$ the whole shell model spectrum is exactly reproduced by complex MONSTER using any complex HFB transformation $F$. This is to be expected, since ${ }^{20} \mathrm{~F}$ belongs to the same isospin multiplet like ${ }^{20} \mathrm{Ne} .{ }^{20} \mathrm{Ne}$ encloses states of all isospins $T=0,1,2$, ${ }^{20} \mathrm{~F}$ only the ones with isospins $T=1$ and 2 . This provides another stringent test for the numerics. The largest deviation relative to the shell model ground state energy was found for ${ }^{24} \mathrm{Na}: 2.21 \%$. ${ }^{24} \mathrm{Na}$ is a typical example for which one should better choose different HFB transformations for states with different spins, instead of using one transformation for all states. If on uses the HFB transformation of the $0^{+}$VAMPIR solution for the low spin states $0^{+}$to $3^{+}$and the transformation $\mathrm{F}\left(4^{+}\right)$ for the higher spins, the average deviation of the yrast states $0^{+}$to $5^{+}$can be reduced to $995 \mathrm{keV}$ or $1.26 \%$ of the shell model ground state energy. The possibility to choose different HFB transformations for different spins should always be considered, since we found that the complex MONSTER energies are quite sensitive on the underlying HFB transformation in contrast to the real case [16].

The average deviations of the real MONSTER are always bigger than for the complex approach, apart from ${ }^{24} \mathrm{Na}$, where both are of similar size. But this could be improved by using several HFB transformations in the complex case. As far as the higher excited states are concerned, the complex MONSTER is always superior to the real one, since it is able to describe many more states.

Compared to the even-even case the deviations obtained by complex MONSTER are somewhat bigger for each mass number. It is known that the structure of odd-odd nuclei is generally more complicated. More types of couplings contribute significantly. This seems to be reflected in our results. But also for the odd-odd case the exact spectra are over all rather well approximated by the MONSTER approach.

In fig. 3 the energy spectra of ${ }^{22} \mathrm{Na}$ calculated with the complex and the real MONSTER are plotted up to spin 7 and an energy of $-52 \mathrm{MeV}$. For comparison the lowest shell model energies for each spin are presented. In the real case, MONSTER is built on the HFB transformation determined for the $0^{+}$VAMPIR solution in ${ }^{20} \mathrm{Ne}$. This gave the best agreement with the shell model. The most bound complex VAMPIR solution in this nucleus is the one for the $1^{+}$yrast state. Complex MONSTER on top of the corresponding HFB transformation corrects then the sequence of the yrast states to the one of the exact calculation, where the ground state has spin $3^{+}$. The largest deviation of a complex MONSTER yrast state energy from the shell model value occurs for the $0^{+}$yrast state : $362 \mathrm{keV}$, the smallest for $7^{+}: 83 \mathrm{keV}$. Complex MONSTER shows also for the higher lying states an excellent agreement with the energies of the exact shell model and yields a significant improvement on the real MONSTER. 
Finally, as an example for the heavier odd-odd nuclei, fig. 4 displays the calculated energies of ${ }^{30} \mathrm{P}$. In this figure two real MONSTER calculations are presented, the ones yielding the best and the worst agreement with the result of the exact diagonalization. The former has been got by using the HFB transformation for the $0^{+}$ VAMPIR solution in ${ }^{28} \mathrm{Si}$, the latter by the HFB transformation for the $0^{+}$VAMPIR solution in ${ }^{32} \mathrm{~S}$. The lowest complex VAMPIR solution in ${ }^{30} \mathrm{P}$ was found to be the one with spin $0^{+}$. Its HFB transformation was used in the complex calculation. The complex and the real MONSTER results are plotted up to $\sim 11 \mathrm{MeV}$ above the shell model ground state for spins $0^{+}$to $5^{+}$. For each spin the lowest energies of the exact diagonalization are given. Complex MONSTER reproduces the general trends of the spectrum rather well. The largest deviation of the yrast state energies is found for spin $2^{+}$and amounts to $1579 \mathrm{keV}$ or $1.01 \%$ of the shell model ground state energy, the smallest, $1157 \mathrm{keV}$ or $0.74 \%$, for spin $0^{+}$. The complex calculation shows a clear improvement on both real ones, where much less states are described.

\subsection{Odd mass nuclei}

As described in section (2.3), in the case of odd mass nuclei the complex MONSTER spectrum has to be built on a HFB transformation determined for a neighbouring even mass nucleus. We proceeded here in the following way. We calculated the shell model spectra of several of those odd mass nuclei, which are neighbouring the even mass nuclei discussed in sections (3.1) and (3.2). Then we used the HFB transformations, which we had already determined, to built up the MONSTER one-quasiparticle configuration spaces. Accordingly, for the mass parameter of the Chung-Wildenthal force, we took always the mass of the even mass nucleus of which the HFB transformation was used. The energy spectra of ${ }_{10}^{21} \mathrm{Ne},{ }_{11}^{23} \mathrm{Na},{ }_{12}^{25} \mathrm{Mg},{ }_{13}^{27} \mathrm{Al}$ and ${ }_{14}^{29} \mathrm{Si}$ have been calculated. For ${ }^{21} \mathrm{Ne}$ the HFB transformations of the deepest VAMPIR solutions in ${ }^{22} \mathrm{Ne}$ and ${ }^{22} \mathrm{Na}$ have been used. The other odd mass nuclei with mass number A have been calculated always in four different ways : using the HFB transformation for the deepest VAMPIR solution of the even-even neighbour with mass $A-1$, the one of the even-even neighbour with mass $A+1$ and the ones of the odd-odd neighbours with $\mathrm{A}-1$ and $\mathrm{A}+1$. Thus, e.g., ${ }^{29} \mathrm{Si}$ has been calculated using the mean fields of ${ }^{28} \mathrm{Si},{ }^{30} \mathrm{Si},{ }^{28} \mathrm{Al}$ and ${ }^{30} \mathrm{P}$. In the case of real MONSTER only the mean fields of the two neighbouring even-even nuclei are available. Both have been used for the real calculations.

The MONSTER configuration space for the calculation of odd mass nuclei is much smaller than the one for the even mass nuclei, because there are much less one-

quasiparticle than two-quasiparticle excitations. For complex MONSTER in the $s d$ shell there are 12 configurations available for states with spin $I=1 / 2,20$ for $I=3 / 2$ and always 24 for states with higher spins. The real MONSTER provides even only 
3 configurations for $I=1 / 2$ states, 5 for $I=3 / 2$, and always 6 for all higher spin states.

Let us first turn our attention to ${ }^{21} \mathrm{Ne}$. It is the lightest odd mass nucleus which we have calculated. For this nucleus there are in total 1935 shell model configurations. More precisely, there are $199 I=1 / 2,341 I=3 / 2,400 I=5 / 2,368 I=7 / 2,287$ $I=9 / 2,183 I=11 / 2,100 I=13 / 2$, $41 I=15 / 2,14 I=17 / 2$, and $2 I=19 / 2$ states. Fig. 5 shows for each spin the lowest energies resulting from the complete shell model diagonalization up to spin $I=11 / 2$. In addition the results of two complex MONSTER calculations are displayed up to an energy of $-38 \mathrm{MeV}$. One calculation was done by using the mean field of the $0^{+}$ground state for ${ }^{22} \mathrm{Ne}$, the other is built on the mean field of the deepest VAMPIR solution for ${ }^{22} \mathrm{Na}$, i.e., the $1^{+}$solution. In fig. 5 also the result of a real MONSTER calculation is plotted for comparison. It is built on the HFB transformation determined for the $0^{+}$ground state of ${ }^{22} \mathrm{Ne}$ by a real VAMPIR calculation. The obtained agreement between both complex MONSTER results and the shell model is very good. For the calculation built on ${ }^{22} \mathrm{Ne}$ the average deviation of the yrast state energies from the exact ones is $490 \mathrm{keV}$. That is $1.03 \%$ of the shell model ground state energy, which is $-47.753 \mathrm{MeV}$. The largest deviation occurs for the $11 / 2$ state and amounts to $664 \mathrm{keV}$ or $1.39 \%$ of the shell model ground state energy, the smallest for spin $5 / 2$, and is $256 \mathrm{keV}$ or $0.54 \%$. The calculation using the mean field of ${ }^{22} \mathrm{Na}$ gives an average deviation of $463 \mathrm{keV}$ or $0.97 \%$ measured relative to the shell model ground state energy. The largest deviation, $653 \mathrm{keV}$ or $1.37 \%$, was found for the $1 / 2$ state, the smallest, $308 \mathrm{keV}$ or $0.64 \%$, for the $7 / 2$ yrast state. Thus, the mean field of the $1^{+}$yrast state of ${ }^{22} \mathrm{Na}$ is on average slightly better suited for the description of the yrast states of ${ }^{21} \mathrm{Ne}$ than that of the $0^{+}$ground state of ${ }^{22} \mathrm{Ne}$. Except for the spin $1 / 2$ and $5 / 2$ yrast states, it yields deeper bound states. Also the excited states obtained by this calculation display the bigger binding energies. The real MONSTER gives for the yrast state energies an average deviation of $576 \mathrm{keV}$ or $1.21 \%$ from the shell model ones. The $1 / 2$ state shows the largest deviation : $704 \mathrm{keV}$ or $1.47 \%$, the $9 / 2$ state the smallest : $467 \mathrm{keV}$ or $0.98 \%$. For most of the yrast states both complex MONSTER calculations yield more binding than the real ones, as it is expected. However, e.g., for the $9 / 2$ yrast state the real MONSTER yielded the deepest energy. The complex results, built on the mean fields of ${ }^{22} \mathrm{Na}$ and ${ }^{22} \mathrm{Ne}$, are higher by $16 \mathrm{keV}$ and $114 \mathrm{keV}$, respectively. This indicates that in the complex case, the mean field determined for a low spin may not be well suited for the description of high spin states.

As example for an heavier odd mass nucleus, we present the energy spectra of ${ }^{29} \mathrm{Si}$ obtained by using the mean fields of the mass 28 systems. The number of shell model states in this nucleus is huge compared to the number of MONSTER configurations available. In total this nucleus has 80112 shell model states. 5638 of these states have spin $I=1 / 2,10176 I=3 / 2,12877 I=5 / 2,13450 I=7 / 2,12240 I=9 / 2$, $9835 I=11 / 2,7053 I=13 / 2,4469 I=15 / 2,2502 I=17 / 2,1197 I=19 / 2,485$ 
$I=21 / 2,152 I=23 / 2,35 I=25 / 2$, and 3 are spin $I=27 / 2$ states. For each spin up to spin $9 / 2$ the lowest energies resulting from the exact shell model calculation are shown in fig. 6. The obtained MONSTER spectra are plotted up to an energy of $\sim 10 \mathrm{MeV}$ above the shell model ground state energy of $-147.020 \mathrm{MeV}$. One complex MONSTER spectrum is obtained by using the HFB transformation of the VAMPIR solution for the $0^{+}$ground state in ${ }^{28} \mathrm{Si}$, the other uses the transformation of the $0^{+}$VAMPIR solution in ${ }^{28} \mathrm{Al}$. The calculation on top of the mean field of the even-even nucleus shows an average deviation of the yrast state energies from the exact ones of $1373 \mathrm{keV}$ or $0.93 \%$ in terms of percentage of the shell model ground state energy. The yrast state with spin $9 / 2$ shows the largest deviation, $1575 \mathrm{keV}$ or $1.07 \%$, the $7 / 2$ yrast state the smallest, $1291 \mathrm{keV}$ or $0.88 \%$. The average deviation obtained by using the mean field of the odd-odd nucleus is somewhat bigger, namely $1912 \mathrm{keV}$ or $1.30 \%$. The largest and smallest deviations, which occur, are $2172 \mathrm{keV}$ or $1.48 \%$ and $1607 \mathrm{keV}$ or $1.09 \%$, respectively. The general trend of the shell model spectrum is reproduced by both complex calculations. In fig. 6 in addition also a real MONSTER spectrum is shown. It was obtained by using the HFB transformation of the $0^{+}$VAMPIR solution in ${ }^{28} \mathrm{Si}$. This spectrum yielded a better agreement with the shell model result than the real calculation on top of the mean field for ${ }^{30} \mathrm{Si}$. For the displayed real MONSTER spectrum the average deviation of the yrast state energies from the exact ones is $1973 \mathrm{keV}$ or $1.34 \%$. The largest deviation occurs for the $1 / 2$ yrast state, $2286 \mathrm{keV}$ or $1.55 \%$, the smallest for $7 / 2,1640 \mathrm{keV}$ or $1.12 \%$. The complex MONSTER calculation on top of the mean field of ${ }^{28} \mathrm{Si}$ shows a clear improvement over this best real result.

For all the considered odd mass nuclei, apart from ${ }^{21} \mathrm{Ne}$, we found that by using the HFB transformation of a neighbouring even-even nucleus, a better agreement between the complex MONSTER result and the shell model could be achieved than by using the mean field of an odd-odd neighbour. In general the odd mass spectra on top of a mean field of a neighbouring even-even nucleus approximated the shell model results well (more details can be found in [20]). The only exceptions were the spectrum of ${ }^{23} \mathrm{Na}$ obtained by using the HFB transformation of the $0^{+}$VAMPIR solution for ${ }^{22} \mathrm{Ne}$ and the spectrum of ${ }^{25} \mathrm{Mg}$ with the transformation for ${ }^{26} \mathrm{Mg}$. These two calculations gave a rather poor agreement with the exact result. In both cases it could be much improved by using the HFB transformation of ${ }^{24} \mathrm{Mg}$. Thus, in the odd mass case the choice of the neighbour can be crucial. One should always try several ones to find the mean field, of which the structure is best suited for the odd mass nucleus under consideration.

Finally we investigated for the odd mass case the dependence of the complex MONSTER energies on the spin of the mean field determined by the preceding VAMPIR calculation. For ${ }^{21} \mathrm{Ne}$ we built always the full MONSTER spectrum on each of the yrast solutions of ${ }^{22} \mathrm{Ne}$ obtained by complex VAMPIR. The same was done for real MONSTER. Naturally here only mean fields for even spin states are available. 
Fig. 7 shows a typical result of this investigation. The energies of the five lowest $3 / 2$ states of ${ }^{21} \mathrm{Ne}$ are plotted in dependence on the spin used in the preceding VAMPIR calculation for ${ }^{22} \mathrm{Ne}$. For comparison, the leftmost column presents the energies of the three lowest $3 / 2$ shell model states. Solid lines denote the results of the complex MONSTER, dotted ones those of the real counterpart. It can be seen that real MONSTER displays only a weak dependence on the spin of the underlying HFB transformations. For the complex MONSTER this is also true if the VAMPIR transformations of the low spin mean fields, $0^{+}, 2^{+}$and $4^{+}$, are considered. The mean fields of the higher spin states are insufficient for the description of the $3 / 2$ states. The poor description of the $3 / 2$ states using the VAMPIR transformations of the low odd spins, $1^{+}, 3^{+}$and $5^{+}$, is due to the fact that those yrast states are dominated by structures which are missing in a complex VAMPIR calculation [16].

The same behaviour as for the $3 / 2$ states was also found for most of the spins of

${ }^{21} \mathrm{Ne}$. Only for the very high spins, where the number of MONSTER configuration is of similar size as the number of shell model states, the dependence becomes small. When the number of MONSTER configurations is bigger than the number of shell model states, the resulting energies are independent of the underlying transformation.

This investigation confirms what we have found already for the even mass case 16]. The complex HFB transformation shows a much stronger dependence on the spin of the underlying VAMPIR calculation than the real one. It means that the structure of the complex mean field differs more from spin to spin. The choice of the spin of the underlying VAMPIR calculation is important in the complex description of odd mass nuclei, too.

\section{Summary}

A recently developed nuclear structure approach, the complex MONSTER, has been studied. The approach is the newest member of a group of models, which are based on variational methods. This group can be divided into two subgroups, one consisting of the VAMPIR models, the other of the MONSTER approaches. Both are designed for large scale nuclear structure calculations.

The models of the VAMPIR family use symmetry-projected HFB quasiparticle vacua as test wavefunctions. The underlying HFB transformations are determined by variation. By construction, these models can only be used to describe the lowest few states of a certain symmetry. In the first numerically realized VAMPIR models the HFB transformations were rather restricted : time-reversal invariance and axial symmetry were required, parity and proton-neutron mixing were neglected and 
only real HFB transformation coefficients were allowed. In the newest models most restrictions are removed and essentially complex HFB transformations are allowed. Only time reversal and axiality are kept.

In the MONSTER approaches the nuclear wavefunctions are expanded around a VAMPIR solution for the ground or an yrast state. The spectrum of excited states is obtained by diagonalizing the Hamiltonian in the space of the VAMPIR solution and all symmetry-projected two-quasiparticle configurations with respect to it. These models are therefore suited for problems, where a complete set of excitations with respect to a particular transition operator is needed. Till lately the MONSTER approach was restricted to the use of real VAMPIR solutions. In a recent work it was generalized. Now complex HFB transformations can be used to build up the configuration space. Then more nucleon correlations are considered already in the mean field and the configuration space becomes much larger. Consequently a more detailed description of nuclear spectra is expected by this complex MONSTER approach. Furthermore one can use HFB transformations determined for the particular system under consideration not only for even-even systems, as in the real approach, but also for odd-odd systems. Only for odd mass nuclei, which are described in the one-quasiparticle approximation, one still has to use the mean field of a neighbouring nucleus, which now may be even-even or odd-odd. To get insight into the ability of this complex approach a systematic investigation was performed in this work.

We presented first results of an application of the complex MONSTER approach to several nuclei in $s d$ shell. This basis, which is rather tiny for a MONSTER calculation, has been chosen to enable a comparison with complete shell model configuration mixing calculations. The investigated even-even and odd-odd nuclei have masses between $A=20$ and 30, the odd mass nuclei reach from $A=21$ to 29 . Thus, quite light systems with only a few valence nucleons are considered as well as heavier systems, up to ${ }^{28} \mathrm{Si}$ with the biggest number of shell model configurations in $s d$ shell.

For the even-even nuclei the complex MONSTER yielded an excellent agreement with the exact shell model approach. The largest mean deviation of the yrast state energies from the shell model result, averaged over the states with spins 0 to 5 , was found for ${ }^{26} \mathrm{Mg}$ and amounts only to $1.43 \%$ of its shell model ground states energy. Concerning the odd-odd systems it was demonstrated that complex MONSTER reproduced the shell model spectrum of ${ }^{20} \mathrm{~F}$ exactly. ${ }^{24} \mathrm{Na}$ showed the largest average deviation of the energies of the yrast states with spins 0 to 5 from the shell model ones, namely $2.21 \%$ of the ground state energy. The average deviations of the oddodd systems were slightly bigger than the ones of the even-even systems with the same mass number. But in general the complex MONSTER yielded a very good approximation to the shell model result also for odd-odd nuclei. For both even-even 
and odd-odd nuclei a clear improvement on the previous real approach was proved.

For odd mass nuclei it was found that the description depends much on the underlying mean field. By choosing a suitable mean field a good agreement with the shell model could be achieved for all investigated nuclei. As examples the results of ${ }^{21} \mathrm{Ne}$ and ${ }^{29} \mathrm{Si}$ were presented. For ${ }^{21} \mathrm{Ne}$, using the HFB transformation of the $1^{+}$ VAMPIR solution in ${ }^{22} \mathrm{Na}$, the deviation of the yrast states energies, averaged over spins $1 / 2$ to $11 / 2$, was $0.97 \%$ of its shell model ground state energy. For ${ }^{29} \mathrm{Si}$, with the mean field determined for the ground state of ${ }^{28} \mathrm{Si}$, the average deviation of the yrast states energies for spins $1 / 2$ to $9 / 2$ amounted to $0.93 \%$ relative to the ground state energy. Again the complex approach achieved better agreement with the shell model than the real one.

Already the previous real MONSTER has been applied successfully to the description of nuclear structure phenomena in various mass regions, e.g., in the mass 130 region [21] 22]. The present investigation has clearly shown that the new complex approach is even superior. So we expect that the complex MONSTER approach will develop as a more powerful tool for nuclear structure studies in large model spaces.

Up to now only the energies calculated by the different models have been compared. It will be interesting to look also for other observables, like transition strengths up to high excitation energies, and to analyze the wavefunctions. This is planned for the future. Finally we would like to mention that the description of nuclear wavefunctions by VAMPIR and MONSTER could be improved by removing all restrictions imposed on the HFB transformation. The development of such approaches allowing the most general HFB transformations is in progress, too.

We thank Prof. Dr. Herbert Müther for performing the shell model calculations with the Glasgow code. Furthermore this work was partly supported by the Graduiertenkolleg "Struktur und Wechselwirkung von Hadronen und Kernen" (DFG, Mu $705 / 3)$. 


\section{References}

[1] P.J. Brussard and P.W.M. Glaudemans, Shell Model Applications in Nuclear Spectroscopy (North-Holland, Amsterdam, 1977).

[2] J.B. McGrory, B.H. Wildenthal, Ann. Rev. Nucl. Part. Sci. 30, 383 (1980).

[3] B.A. Brown and B.H. Wildenthal, Ann. Rev. Nucl. Part. Sci. 38, 29 (1988).

[4] K.W. Schmid and F. Grümmer, Rep. Prog. Phys. 50, 731 (1987).

[5] D.R. Hartree, Proc. Cambridge Philos. Soc. 24, 89 (1928).

[6] V. Fock, Z. Phys. 61, 126 (1930).

[7] N.N. Bogoliubov, Zk. Eksp. Teor. Fiz. 34, 58 (1958).

[8] P. Ring and P. Schuck, The Nuclear Many Body Problem (Springer, New York/Heidelberg/Berlin, 1980).

[9] K.W. Schmid, F. Grümmer and A. Faessler, Phys. Rev. C 29, 291 (1984).

[10] K.W. Schmid, F. Grümmer and A. Faessler, Nucl. Phys. A431, 205 (1984).

[11] K.W. Schmid, F. Grümmer, M. Kyotoku and A. Faessler, Nucl. Phys. A452, 493 (1986).

[12] K.W. Schmid, R.-R. Zheng, F. Grümmer and A. Faessler, Nucl. Phys. A499, $63(1989)$.

[13] K.W. Schmid, F. Grümmer and A. Faessler, Phys. Rev. C 29, 308 (1984).

[14] K.W. Schmid, F. Grümmer and A. Faessler, Ann. Phys. 180, 1 (1987).

[15] R.-R. Zheng, K.W. Schmid, F. Grümmer and A. Faessler, Nucl. Phys. A494, 214 (1989).

[16] E. Bender, K.W. Schmid and A. Faessler, submitted to Nucl. Phys. A.

[17] H.J. Mang, Phys. Rep. 18, 325 (1975).

[18] F. Ajzenberg-Selove, Nucl. Phys. A460, 1 (1986).

[19] B.H. Wildenthal, Prog. Part. Nucl. Phys. 11, 5 (1983).

[20] E. Bender, PhD Thesis, in preparation.

[21] E. Hammarén, K.W. Schmid, F. Grümmer, A. Faessler and B. Fladt, Nucl. Phys. A437, 1 (1985).

[22] E. Hammarén, K.W. Schmid, F. Grümmer, A. Faessler and B. Fladt, Nucl. Phys. A454, 301 (1986). 


\section{Figure Captions}

Fig. 1 Comparison of the energy spectra of ${ }^{28} \mathrm{Si}$ for spins 0 to 6 as obtained by three different approaches : the shell model configuration mixing approach (SCM), the complex MONSTER (CM) and the real MONSTER (RM). The energy is given relative to the ${ }^{16} \mathrm{O}$ core. For each spin only the lowest few shell model states are presented. The MONSTER results are displayed up to excitation energies of $\sim 14 \mathrm{MeV}$ above the shell model ground state. The complex and the real MONSTER calculation has been built on the corresponding complex and real VAMPIR solution for the $0^{+}$ground state.

Fig. 2 The energies of the yrast states with spin $0^{+}$to $6{ }^{+}$in ${ }^{28} \mathrm{Si}$ as obtained by various approaches : the shell model (SCM), the complex MONSTER (CM) (based on the $0^{+}$solution of complex VAMPIR), the complex VAMPIR (CV), the real MONSTER (RM) (on top of the $0^{+}$solution of real VAMPIR), and the real VAMPIR (RV). In the last approach only the even spin states are accessible. Spin and parity are indicated on the l.h.s. of each level. The energy is given relative to the ${ }^{16} \mathrm{O}$ core.

Fig. 3 Same as Fig. 1, but for the odd-odd nucleus ${ }^{22} \mathrm{Na}$ and up to spin 7 . Each MONSTER spectrum is shown up to $7.5 \mathrm{MeV}$ above the shell model ground state. The complex MONSTER was built on the complex VAMPIR solution obtained for the $1^{+}$yrast state, the real MONSTER was based on the real VAMPIR solution for the $0^{+}$ground state of ${ }^{20} \mathrm{Ne}$.

Fig. 4 Same as Fig. 1, but for the odd-odd nucleus ${ }^{30} \mathrm{P}$ and up to spin 5 . The results of two real MONSTER calculations are displayed. Each MONSTER spectrum is shown up to $11 \mathrm{MeV}$ above the shell model ground state. The complex MONSTER was built on the complex VAMPIR solution obtained for the $0^{+}$yrast state. One real MONSTER calculation has been based on the VAMPIR transformation for the $0^{+}$ground state of ${ }^{28} \mathrm{Si}$, the other on the one of the $0^{+}$ground state of ${ }^{32} \mathrm{~S}$.

Fig. 5 Same as Fig. 1, but for the odd mass nucleus ${ }^{21} \mathrm{Ne}$ and for spins $1 / 2$ to $11 / 2$. The results of two complex MONSTER calculations are displayed. Each MONSTER spectrum is shown up to $-38 \mathrm{MeV}$. One complex MONSTER calculation has been based on the complex VAMPIR transformation for the $0^{+}$ground state of ${ }^{22} \mathrm{Ne}$, the other was using the one for the $1^{+}$yrast state of ${ }^{22} \mathrm{Na}$. The real MONSTER calculation was built on the real VAMPIR solution for the $0^{+}$ground state of ${ }^{22} \mathrm{Ne}$.

Fig. 6 Same as Fig. 1, but for the odd mass nucleus ${ }^{29} \mathrm{Si}$ and for spins $1 / 2$ to 9/2. The results of two complex MONSTER calculations are displayed. Each MONSTER spectrum is shown up to $10 \mathrm{MeV}$ above the shell model ground state. One complex MONSTER calculation has been based on the complex 
VAMPIR transformation for the $0^{+}$ground state of ${ }^{28} \mathrm{Si}$, the other was using the one for the $0^{+}$yrast state of ${ }^{28} \mathrm{Al}$. The real MONSTER calculation was built on the real VAMPIR solution for the $0^{+}$ground state of ${ }^{28} \mathrm{Si}$.

Fig. 7 The five lowest $3 / 2^{+}$MONSTER states of ${ }^{21} \mathrm{Ne}$ are plotted versus the spin of the yrast state in ${ }^{22} \mathrm{Ne}$, of which the VAMPIR transformation was used in each calculation. Solid lines refer to the complex MONSTER, dotted lines to the real MONSTER results. In the latter case only transformations for even spin values are available. For comparison the lowest three $3 / 2^{+}$shell model (SCM) energies are also given. The energy is given relative to the ${ }^{16} \mathrm{O}$ core. 


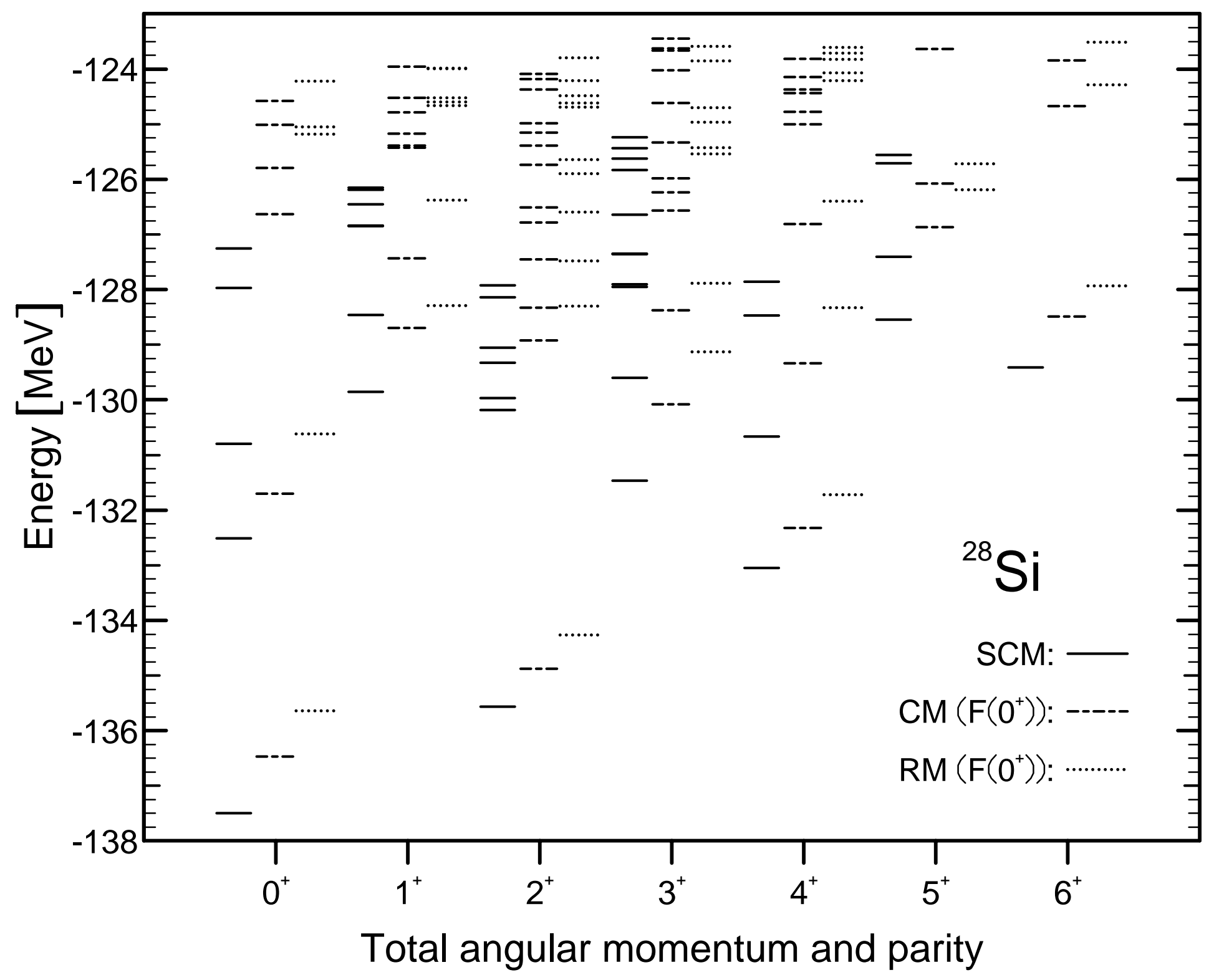


Table 1:

A comparison of the deviations of the yrast state energies calculated with complex and real MONSTER from the exact shell model energies is provided for the considered even-even nuclei. In addition the minimum, the maximum and an average number of shell model states are shown.

\begin{tabular}{||c||c|c|c|c|c|c||}
\hline \hline & ${ }^{20} \mathrm{Ne}$ & ${ }^{22} \mathrm{Ne}$ & ${ }^{24} \mathrm{Mg}$ & ${ }^{26} \mathrm{Mg}$ & ${ }^{28} \mathrm{Si}$ & ${ }^{30} \mathrm{Si}$ \\
\hline \hline $\mathrm{A} 1 \mathrm{CM}$ & 0 & 174 & 560 & 1529 & 1113 & 972 \\
\cline { 2 - 7 } & 390 & 510 & 2145 & 2064 & 1791 & 1798 \\
\hline \hline $\mathrm{SCM}_{\text {gs }}$ & -41.412 & -58.709 & -88.610 & -107.144 & -137.495 & -156.536 \\
\hline \hline $\mathrm{A} 2 \stackrel{\mathrm{CM}}{\mathrm{R}}$ & $0 \%$ & $0.30 \%$ & $0.63 \%$ & $1.43 \%$ & $0.81 \%$ & $0.62 \%$ \\
\hline \hline $\mathrm{N}_{\text {min }}$ & $46\left(0^{+}\right)$ & $216\left(0^{+}\right)$ & $1161\left(0^{+}\right)$ & $1968\left(0^{+}\right)$ & $3372\left(0^{+}\right)$ & $1968\left(0^{+}\right)$ \\
\hline $\mathrm{N}_{\text {max }}$ & $143\left(2^{+}\right)$ & $798\left(3^{+}\right)$ & $4968\left(3^{+}\right)$ & $8733\left(3^{+}\right)$ & $15385\left(3^{+}\right)$ & $8733\left(3^{+}\right)$ \\
\hline $\mathrm{N}_{\text {aver }}$ & 98 & 596 & 3720 & 6557 & 11583 & 6557 \\
\hline \hline
\end{tabular}

A1: average deviation of the energies of the yrast states $0^{+}$to $5^{+}$calculated with complex (CM) and real (RM) MONSTER from the shell model energies in keV.

$\mathrm{SCM}_{g s}$ : shell model ground state energy in $\mathrm{MeV}$.

A2: $\quad$ same as A1 but now in percentage of the shell model ground state energy.

$\mathrm{N}_{\text {min }}$ : minimum number of shell model states states per spin and corresponding spin.

$\mathrm{N}_{\text {max }}$ : maximum number of shell model states states per spin and corresponding spin.

$\mathrm{N}_{\text {aver }}$ : number of shell model states per spin averaged over spins $0^{+}$to $5^{+}$. 


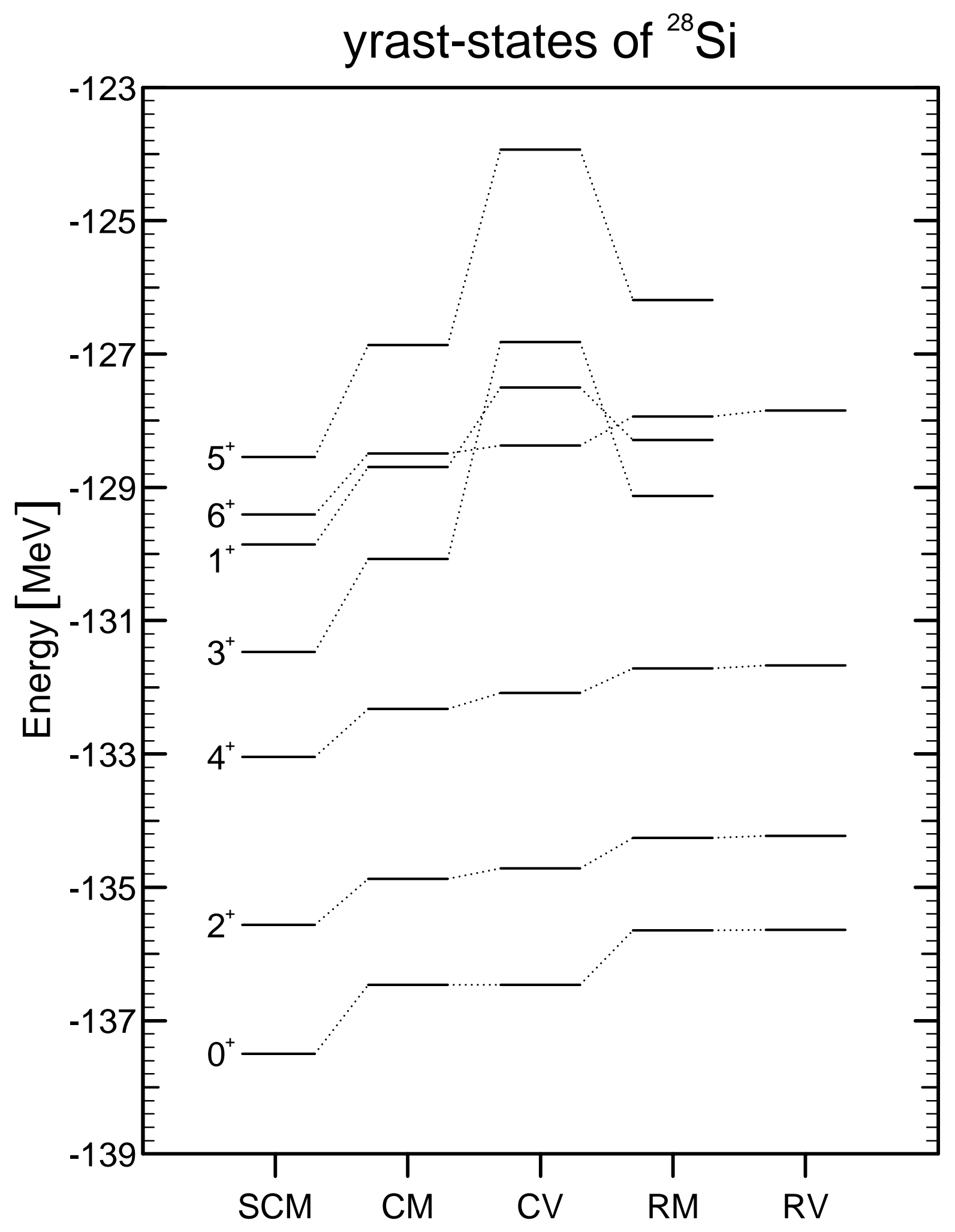


Table 2:

Same as table 1 but for the considered odd-odd nuclei.

\begin{tabular}{|c|c|c|c|c|c|c|c|}
\hline & ${ }^{20} \mathrm{~F}$ & ${ }^{22} \mathrm{Na}$ & ${ }^{24} \mathrm{Na}$ & ${ }^{26} \mathrm{Al}$ & ${ }^{28} \mathrm{Al}$ & ${ }^{30} \mathrm{P}$ \\
\hline \multirow{2}{*}{ A1 } & $\mathrm{CM}$ & 0 & 227 & 1749 & 1616 & 1556 & 1345 \\
\hline & $\mathrm{RM}$ & 123 & 876 & 1754 & 2151 & 2173 & 2016 \\
\hline \multirow{2}{*}{\multicolumn{2}{|c|}{$\mathrm{SCM}_{g s}$}} & -31.216 & -59.446 & -78.991 & -107.182 & -128.144 & -157.066 \\
\hline & & $2^{+}$ & $3^{+}$ & $4^{+}$ & $5^{+}$ & $2^{+}$ & $1^{+}$ \\
\hline \multirow{2}{*}{$\mathrm{A} 2$} & $\mathrm{CM}$ & $0 \%$ & $0.38 \%$ & $2.21 \%$ & $1.51 \%$ & $1.21 \%$ & $0.86 \%$ \\
\hline & RM & $0.39 \%$ & $1.47 \%$ & $2.22 \%$ & $2.01 \%$ & $1.70 \%$ & $1.28 \%$ \\
\hline \multirow{2}{*}{$\mathrm{F}$} & $\mathrm{CM}$ & any & $\mathrm{F}\left(1^{+}\right)$ & $\mathrm{F}\left(4^{+}\right)$ & $\mathrm{F}\left(0^{+}\right)$ & $\mathrm{F}\left(0^{+}\right)$ & $\mathrm{F}\left(0^{+}\right)$ \\
\hline & $\mathrm{RM}$ & $\mathrm{F}\left({ }^{22} \mathrm{Ne}\right)$ & $\mathrm{F}\left({ }^{20} \mathrm{Ne}\right)$ & $\mathrm{F}\left({ }^{24} \mathrm{Mg}\right)$ & $\mathrm{F}\left({ }^{28} \mathrm{Si}\right)$ & $\mathrm{F}\left({ }^{28} \mathrm{Si}\right)$ & $\mathrm{F}\left({ }^{28} \mathrm{Si}\right)$ \\
\hline \multicolumn{2}{|c|}{$\mathrm{N}_{\min }$} & $25\left(0^{+}\right)$ & $287\left(0^{+}\right)$ & $836\left(0^{+}\right)$ & $2533\left(0^{+}\right)$ & $2533\left(0^{+}\right)$ & $2533\left(0^{+}\right)$ \\
\hline \multicolumn{2}{|c|}{$\mathrm{N}_{\max }$} & $87\left(2^{+}\right)$ & $1164\left(3^{+}\right)$ & $3664\left(3^{+}\right)$ & $11674\left(3^{+}\right)$ & $11674\left(3^{+}\right)$ & $11674\left(3^{+}\right)$ \\
\hline \multicolumn{2}{|c|}{$\mathrm{N}_{\text {aver }}$} & 61 & 855 & 2721 & 8745 & 8745 & 8745 \\
\hline
\end{tabular}

In addition to the shorthand notations of table 1, we have used:

F: the underlying HFB transformation $F$ is indicated. For the complex MONSTER (CM) the spin of the corresponding VAMPIR solution is given. In the real case (RM) the neighbouring even-even nucleus is shown, which has been used to determine the HFB transformation.

$\mathrm{SCM}_{g s}$ : contains in addition to the energy, as in table 1, also the shell model ground state spin. 


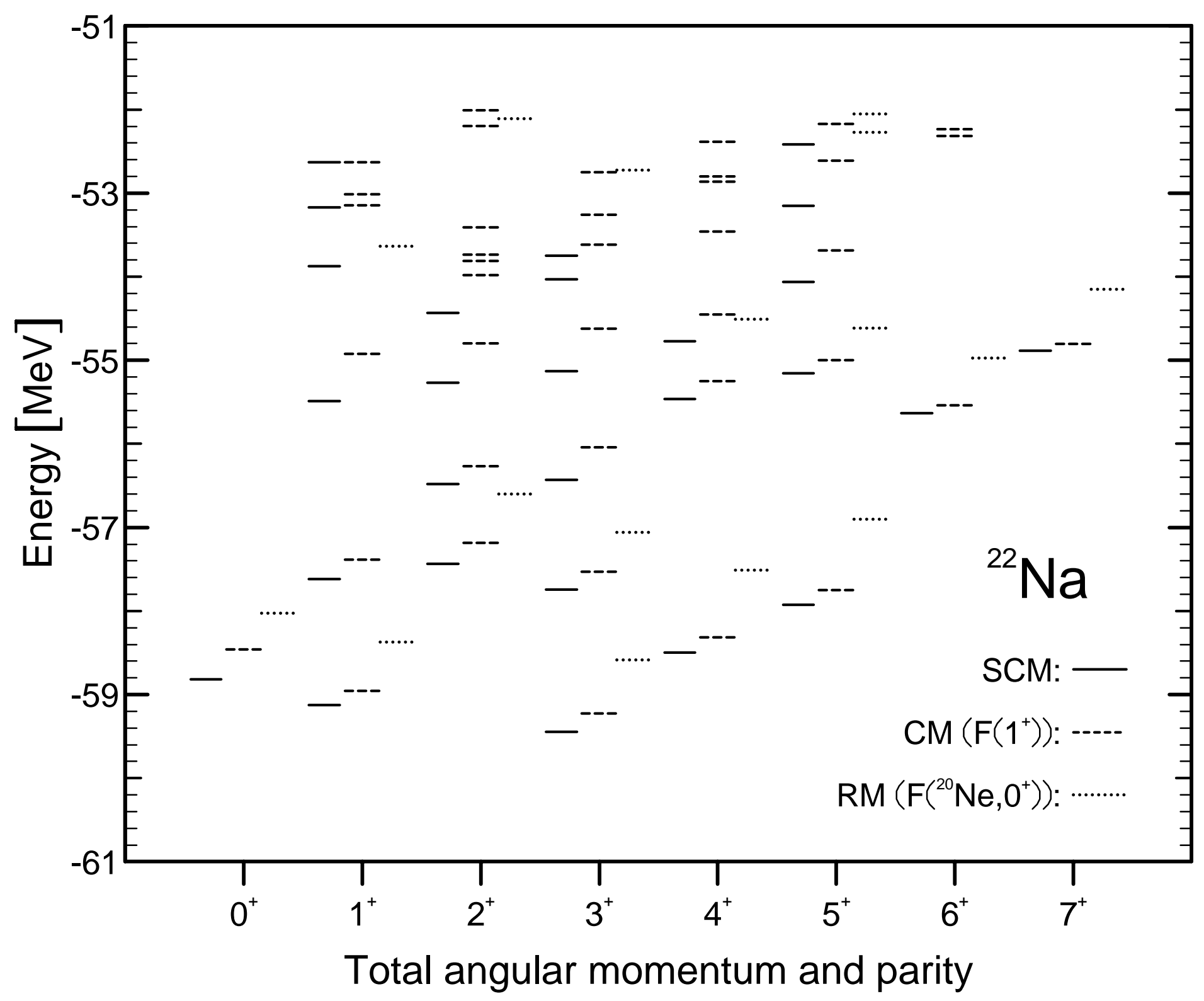




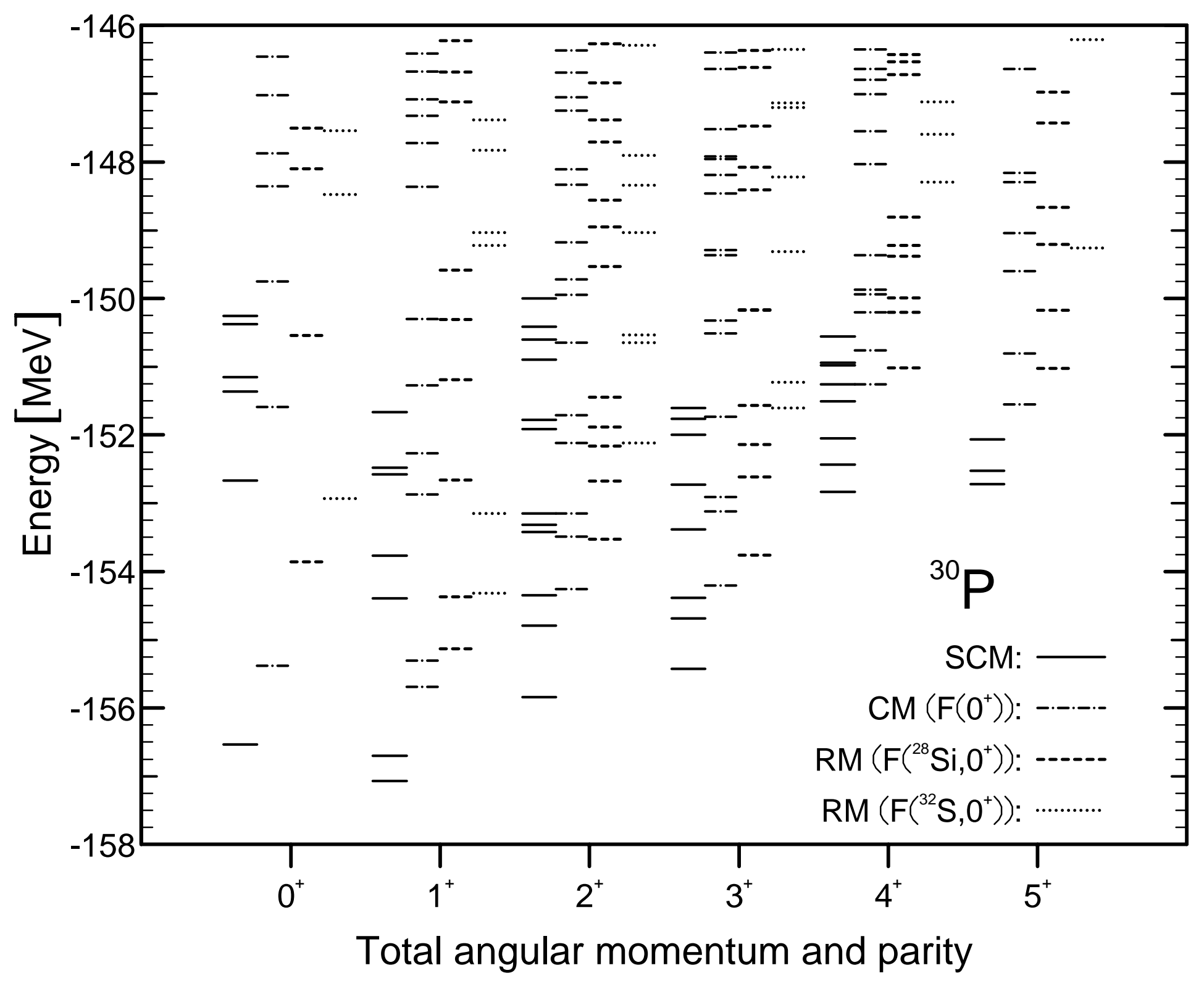









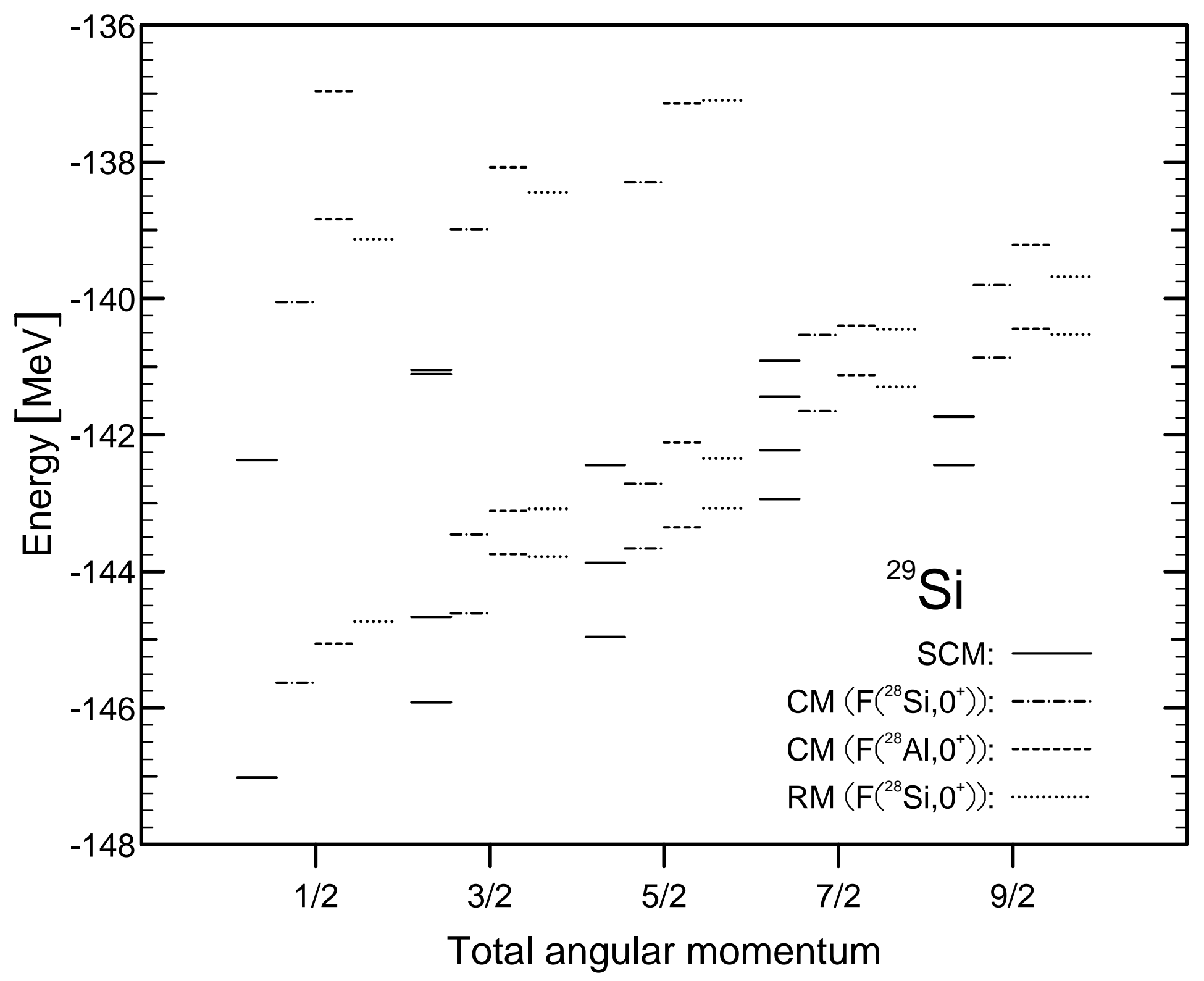




\section{$3 / 2^{+}$states of ${ }^{21} \mathrm{Ne}$}

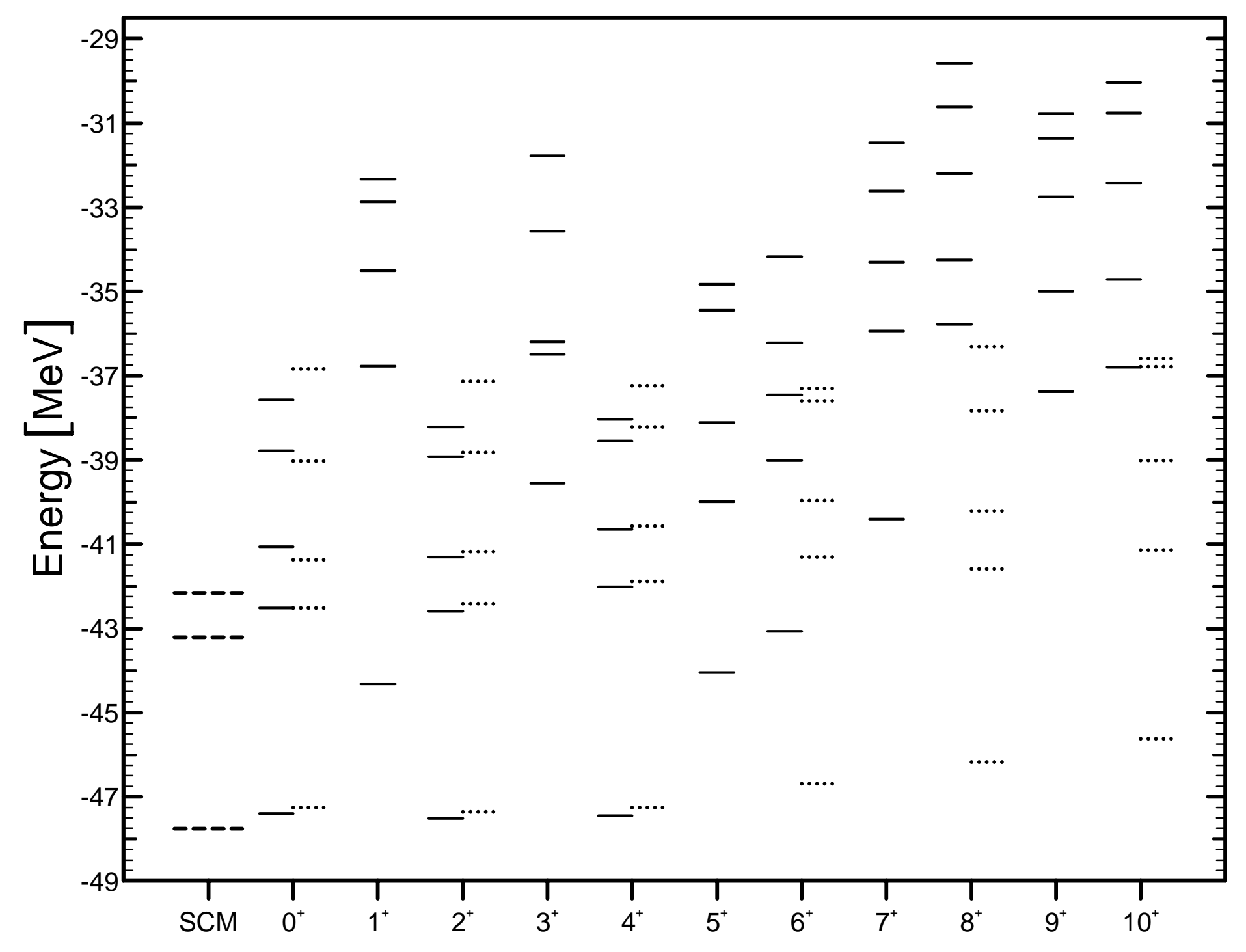

